\title{
Modification of Concrete Damaged Plasticity model. Part II: Formulation and numerical tests
}

\author{
Inez Kamińska ${ }^{1}$, and Aleksander Szwed ${ }^{1, *}$ \\ ${ }^{1}$ Warsaw University of Technology, Al. Armii Ludowej 16, 00-637 Warszawa, Poland
}

\begin{abstract}
A refined model for elastoplastic damaged material is formulated based on the plastic potential introduced in Part I [1]. Considered model is an extension of Concrete Damaged Plasticity material implemented in Abaqus [2]. In the paper the stiffness tensor for elastoplastic damaged behaviour is derived. In order to validate the model, computations for the uniaxial tests are performed. Response of the model for various cases of parameter's choice is shown and compared to the response of the CDP model.
\end{abstract}

\section{Introduction}

An alteration to the Abaqus' Concrete Damaged Plasticity model is made in attempt to improve its flexibility. The new potential shown in Part I [1] is introduced to the equations of elasticity and plasticity theory in order to derive the stiffness tensor for elastoplastic damaged behavior of material. As mentioned in Part I, the extended model requires input of twelve parameters and four material functions.

To confirm validity of this material model, its consistency with basic experiments has to be proven. The objective of the second part of the present paper is to show and analyze numerical results of the uniaxial loading test for the proposed model concerning improved potential. In order to achieve this goal, a simple forward-Euler algorithm was created using Mathematica [3]. The influence of free parameters $m_{1}, m_{2}, m_{3}$ and $m_{4}$ on the uniaxial test results is considered. The first two parameters indicate the meridians of Lubliner's condition used as asymptotes of the potential, the third one - location of the potential's apex and the last one - the curvature in the vertex region and the zone of transition between potential's asymptotes (near $\sigma_{1}=0$ ). The results of basic tests are compared for various sets of parameters' values.

As it has been noted in Part I, the difference between the new and original Abaqus potential [2] allows more flexibility when it comes to reflecting material properties. Examples of numerical results are shown in comparison to those acquired using the CDP model.

* Corresponding author: a.szwed@il.pw.edu.pl 


\section{Formulation of the model}

Considered elastoplastic damage model for concrete is based on the Lubliner's two-surface failure criterion with isotropic damage described by one scalar damage variable. Plasticity and damage surfaces are the same. The non-associative flow rule is proposed, which is based on the plastic potential described in Part I [1]. Constitutive equations are formulated in the effective stress space [4]. The basic equations and assumptions of the model are as follows.

- The relation between nominal stress tensor and effective stress tensor is:

$$
\hat{\boldsymbol{\sigma}}=\frac{\boldsymbol{\sigma}}{1-d},
$$

where $d$ is a damage parameter.

- Additive decomposition of the tensor of strain rate into elastic $\left(\dot{\boldsymbol{\varepsilon}}_{E}\right)$ and plastic $\left(\dot{\boldsymbol{\varepsilon}}_{P}\right)$ parts and the Hooke's constitutive law for elastic behavior hold:

$$
\dot{\boldsymbol{\varepsilon}}=\dot{\boldsymbol{\varepsilon}}_{E}+\dot{\boldsymbol{\varepsilon}}_{P}, \quad \hat{\boldsymbol{\sigma}}=\mathbf{C} \boldsymbol{\varepsilon}_{E},
$$

where $\mathbf{C}=K \mathbf{I} \otimes \mathbf{I}+2 G\left(\mathbf{1}-\frac{1}{3} \mathbf{I} \otimes \mathbf{I}\right)$ is the stiffness tensor for isotropic elastic undamaged material.

- The dissipation during plastic flow is positive:

$$
D=\hat{\boldsymbol{\sigma}} \dot{\boldsymbol{\varepsilon}}_{P}>0 \text { and } D=\boldsymbol{\sigma} \dot{\boldsymbol{\varepsilon}}_{P}>0 \text { when } \dot{\boldsymbol{\varepsilon}}_{P} \neq \mathbf{0} .
$$

- The yield criterion is Lubliner's condition [5,6]:

$$
f\left(\hat{\boldsymbol{\sigma}}, \varepsilon_{P L, C}\right)= \begin{cases}\frac{1}{1-\alpha}\left(\sqrt{3} \alpha \hat{\xi}+\sqrt{\frac{3}{2}} \hat{r}+\gamma \hat{\sigma}_{1}\right)-\hat{\sigma}_{C}\left(\varepsilon_{P L, C}\right)=0 & \text { when } \hat{\sigma}_{1} \leq 0 \\ \frac{1}{1-\alpha}\left(\sqrt{3} \alpha \hat{\xi}+\sqrt{\frac{3}{2}} \hat{r}+\beta\left(\varepsilon_{P L, C}, \varepsilon_{P L, T}\right) \hat{\sigma}_{1}\right)-\hat{\sigma}_{C}\left(\varepsilon_{P L, C}\right)=0 & \text { when } \hat{\sigma}_{1}>0\end{cases}
$$

where:

$$
\hat{\sigma}_{1}=\frac{1}{\sqrt{3}} \hat{\xi}+\sqrt{\frac{2}{3}} \hat{r} \cos \hat{\theta}, \quad \hat{\theta}=\frac{1}{3} \arccos \left(\frac{\sqrt{6} \operatorname{tr} \hat{\mathbf{s}}^{3}}{\sqrt{\operatorname{tr}^{3} \hat{\mathbf{s}}^{2}}}\right) .
$$

- The plastic potential $\Phi$ is described in Part I. The non-associative flow rule and the consistency condition state:

$$
\dot{\boldsymbol{\varepsilon}}_{P}=\lambda \frac{\partial \Phi(\hat{\boldsymbol{\sigma}})}{\partial \hat{\boldsymbol{\sigma}}}=\lambda \mathbf{p}, \quad \dot{f}\left(\hat{\boldsymbol{\sigma}}, \varepsilon_{P L, C}\right)=0 .
$$

- Plastic hardening is prescribed by the following formulae:

$$
\hat{\sigma}_{C}\left(\varepsilon_{C, P L}\right)=\sigma_{C 0}+E_{P} \varepsilon_{C, I N}\left(\varepsilon_{C, P L}\right), \quad \hat{\sigma}_{T}\left(\varepsilon_{T, P L}\right)=\sigma_{T 0}+E_{P} \varepsilon_{T, I N}\left(\varepsilon_{T, P L}\right),
$$


where $E_{P}$ is a hardening modulus and $\varepsilon_{C, I N}, \varepsilon_{C, P L}, \varepsilon_{T, I N}$ and $\varepsilon_{T, P L}$ are equivalent inelastic and plastic compressive and tensile strains respectively.

- Relationships considering equivalent strains are:

$$
\begin{gathered}
\dot{\varepsilon}_{C, P L}=\left(r^{*}-1\right) \dot{\varepsilon}_{P, M I N}, \quad \dot{\varepsilon}_{T, P L}=r^{*} \dot{\varepsilon}_{P, M A X}, r^{*}=\frac{1}{2} \frac{\hat{\sigma}_{1}+\hat{\sigma}_{2}+\hat{\sigma}_{3}}{\left|\hat{\sigma}_{1}\right|+\left|\hat{\sigma}_{2}\right|+\left|\hat{\sigma}_{3}\right|}, \\
\varepsilon_{T, I N}=\varepsilon_{T, P L}+\frac{d_{T}}{1-d_{T}} \frac{\sigma_{T}}{E_{0}}, \quad \varepsilon_{C, I N}=\varepsilon_{C, P L}+\frac{d_{C}}{1-d_{C}} \frac{\sigma_{C}}{E_{0}},
\end{gathered}
$$

where $\varepsilon_{T, I N}$ and $\varepsilon_{T, P L}$ are equivalent inelastic and plastic tensile strains, $\varepsilon_{C, I N}$ and $\varepsilon_{C, P L}$ equivalent inelastic and plastic compressive strains respectively, $\varepsilon_{P, M A X}$ and $\varepsilon_{P, M I N}$ are the maximum and minimum principal plastic strains, $d_{T}$ and $d_{C}$ are tensile and compressive damage parameters and $E_{0}$ is an initial (undamaged) Young modulus.

- The formulas for damage parameters are:

$$
d=1-\left(1-s_{T} d_{C}\right)\left(1-s_{C} d_{T}\right), d_{T}=H_{T} \varepsilon_{T, I N}, d_{C}=H_{C} \varepsilon_{C, I N},
$$

where $H_{T}$ and $H_{C}$ are material parameters, and $s_{T}$ and $s_{C}$ reflect both material properties and the current effective stress state. See reference [2] for more details.

The Lubliner's yield criterion (4) contains four material constants: values of the compressive yield limit, the compressive biaxial yield limit, the compressive triaxial yield limit and the tensile yield limit of material. To define the plastic potential one needs additionally to determine constants $m_{1}, m_{2}, m_{3}$ and $m_{4}$. The other equations contain four more material constants and four material functions concerning hardening and evolution of damage parameters. Full description of the proposed model of elastoplastic material with isotropic damage requires determining twelve parameters (four of which are chosen

\begin{tabular}{|c|c|c|c|c|}
\hline \multirow{2}{*}{ Equations } & \multirow{2}{*}{$\begin{array}{c}\text { Material } \\
\text { parameters/functions }\end{array}$} & \multicolumn{2}{|c|}{ Number of } & \multirow{2}{*}{ Test } \\
\hline & & const. & fun. & \\
\hline Hooke's law & $E$ and $v$ (or $K$ and $G$ ) & 2 & 0 & uniaxial compression \\
\hline $\begin{array}{l}\text { Lubliner's } \\
\text { yield } \\
\text { condition }\end{array}$ & $\begin{array}{l}\sigma_{T 0}, \sigma_{C 0}, \sigma_{B C} \\
\eta \sigma_{T C}\end{array}$ & 4 & $(2)$ & $\begin{array}{l}\text { uniaxial tension and compression, } \\
\text { biaxial compression, triaxial } \\
\text { compression }\end{array}$ \\
\hline Hardening & $\sigma_{C}\left(\varepsilon_{C, I N}\right), \sigma_{T}\left(\varepsilon_{T, I N}\right)$ & 0 & 2 & uniaxial tension and compression \\
\hline \multirow{2}{*}{$\begin{array}{l}\text { Gradient of } \\
\text { plastic } \\
\text { potential }\end{array}$} & $\begin{array}{l}\sigma_{T 0}, \sigma_{C 0}, \sigma_{B C} \\
\eta \sigma_{T C}\end{array}$ & 4 & 0 & $\begin{array}{l}\text { uniaxial tension and compression, } \\
\text { biaxial compression, triaxial } \\
\text { compression }\end{array}$ \\
\hline & $m_{1}, m_{2}, m_{3}, m_{4}$ & 4 & 0 & $\begin{array}{l}\text { slopes and smoothness - } \\
\text { calibration }\end{array}$ \\
\hline Damage & $\begin{array}{l}d_{C}\left(\varepsilon_{C, I N}\right), d_{T}\left(\varepsilon_{T, I N}\right), \\
\omega_{T}, \omega_{C}\end{array}$ & 2 & 2 & $\begin{array}{l}\text { uniaxial cyclic compression- } \\
\text { tension }\end{array}$ \\
\hline
\end{tabular}
arbitrarily) and four material functions, compare Tab. 1 and $[7,8,9,10]$.

Table 1. Material constants and functions used in the proposed model. 


\section{Stiffness tensor}

The stiffness tensor for the introduced material model $\left(\mathbf{C}^{E P}\right)$ defines the relation between effective stress rate tensor and strain rate tensor:

$$
\dot{\hat{\boldsymbol{\sigma}}}=\mathbf{C}^{E P} \dot{\boldsymbol{\varepsilon}}
$$

To derive a formula for the stiffness tensor one has to investigate several cases. It is essential to consider separately two Lubliner's surfaces (for compression $\hat{\sigma}_{1} \leq 0$, and tension $\hat{\sigma}_{1}>0$ when $0<\theta<\pi / 3$ ), as well as special cases for the tensile and compression meridians only.

Taking into consideration Hooke's law with the additive split of strain rate tensor (2), the flow rule and consistency condition (6) for $0<\theta<\pi / 3$, one arrives at:

$$
\begin{gathered}
\mathbf{C}^{E P}=2 G \mathbf{1}+\{[1 \\
\left.\left.-\frac{\sqrt{3} u(\hat{\theta})}{a_{0}(1-\alpha)}\right] K-\frac{2}{3} G\right\} \mathbf{I} \otimes \mathbf{I}-\frac{1}{a_{0}(1-\alpha)}\left[2 \sqrt{3} K G\left(\frac{w(\hat{\theta})}{\hat{r}} \mathbf{I} \otimes \hat{\mathbf{s}}+\frac{2 v(\hat{\theta})}{\hat{r}^{2}} \mathbf{I} \otimes \hat{\mathbf{s}}^{2}\right)\right. \\
\left.+2 G z(\hat{r})\left(\frac{u(\hat{\theta})}{\hat{r}} \hat{\mathbf{s}} \otimes \mathbf{I}+\frac{2 G w(\hat{\theta})}{\hat{r}^{2}} \hat{\mathbf{s}} \otimes \hat{\mathbf{s}}+\frac{4 G v(\hat{\theta})}{\hat{r}^{3}} \hat{\mathbf{s}} \otimes \hat{\mathbf{s}}^{2}\right)\right]
\end{gathered}
$$

where:

$$
\begin{gathered}
\mu=\left\{\begin{array}{ll}
\beta & \text { when } \hat{\sigma}_{1}>0 \\
\gamma & \text { when } \hat{\sigma}_{1} \leq 0
\end{array},\right.
\end{gathered}, \begin{aligned}
& v(\hat{\theta})=\frac{\mu \sin \hat{\theta}}{\sin 3 \hat{\theta}}, \quad u(\hat{\theta})=(3 \alpha+\mu) K-\frac{4}{3} G v(\hat{\theta}), \quad w(\hat{\theta})=\sqrt{\frac{3}{2}}+\sqrt{\frac{2}{3} \frac{\mu \sin 2 \hat{\theta}}{\sin 3 \hat{\theta}},} \\
& z(\hat{r})=-\frac{\partial \Phi}{\partial \hat{r}}=\hat{r}\left(\frac{\alpha_{1}^{2}\left(f_{s}-f_{2 n}\right)}{f_{1 n}-\beta_{1}}+\frac{\alpha_{2}^{2}\left(f_{s}-f_{1 n}\right)}{f_{2 n}-\beta_{2}}\right)\left(f_{1 n}+f_{2 n}-2 f_{s}\right)^{-1}, \\
& a_{0}=\left\{\begin{array}{l}
y(\hat{r}, \hat{\theta})-\left(1-r^{*}\right) E_{P} p_{\min } \\
y(\hat{r}, \hat{\theta})-\left(1-r^{*}\right)\left(1-\frac{\hat{\sigma}_{1}}{\hat{\sigma}_{T}}\right) E_{P} p_{\min }+r^{*} E_{P} p_{\max } \frac{\hat{\sigma}_{1} \hat{\sigma}_{C}}{\hat{\sigma}_{T}^{2}} \text { when } \hat{\sigma}_{1}>0
\end{array}\right. \\
& y(\hat{r}, \hat{\theta})=\frac{1}{1-\alpha}\left[\sqrt{3}(3 \alpha+\mu) K+2 G z(\hat{r})\left(w(\hat{\theta})+\sqrt{\frac{2}{3}} v(\hat{\theta}) \cos \hat{\theta}\right)\right] .
\end{aligned}
$$

$f_{1 n}, f_{2 n}$ and $f_{s}$ are functions of $\hat{r}[1]$ and $p_{\min }$ is the minimum eigenvalue of gradient $\mathbf{p}$. Plastic multiplier is given by the equation:

$$
\lambda=\frac{1}{a_{0}(1-\alpha)}\left\{\left[(3 \alpha+\mu) K-\frac{4}{3} G v(\hat{\theta})\right] \operatorname{tr} \dot{\boldsymbol{\varepsilon}}+\frac{2 G w(\hat{\theta})}{\hat{r}} \hat{\mathbf{s}}\left[\dot{\boldsymbol{\varepsilon}}+\frac{4 G v(\hat{\theta})}{\hat{r}^{2}} \dot{\boldsymbol{\varepsilon}} \widehat{\mathbf{s}}^{2}\right\} .\right.
$$

The stiffness tensor (11) and the plastic multiplier (12) for the tensile meridian can be determined by finding limits of expressions (11) and (12) for $\theta \rightarrow 0$. Situation is more 
complex for the compression meridian, where the Lubliner's yield condition does not have uniquely defined gradient and one has to assume its form, for example as the DruckerPrager potential gradient or alternatively as the average of gradients of two adjacent patches of the yield surface.

Supposing weak coupling between plasticity and damage:

$$
\dot{\boldsymbol{\sigma}}=(1-d) \dot{\hat{\boldsymbol{\sigma}}}, \quad \text { which entails } \quad \dot{\boldsymbol{\sigma}}=(1-d) \mathbf{C}^{E P} \dot{\boldsymbol{\varepsilon}}=\mathbf{C}^{E P D} \dot{\boldsymbol{\varepsilon}} .
$$

\section{Uniaxial test}

Uniaxial test is a basic experiment determining properties of concrete. It is frequently used to adjust values of the material models' parameters. During the primary stage of loading material is elastic then plastic deformation emerges. Along with plasticity, damage occurs which is observed as the degradation of initial elastic moduli. As the loading reaches a certain point, decrease of volume appears.

In the computations a cyclic change of normal component of stress tensor $\sigma_{X X}$ is assumed. Applied load program is shown on Fig. 1(a). The parameters of the model are as follows: $E=20 \mathrm{GPa}$ and $v=0.2$, which yields $K=11 \mathrm{GPa}$ and $G=8.3 \mathrm{GPa}$; $\sigma_{C 0}=20 M P a, \sigma_{T 0}=2 M P a, \sigma_{B C}=23.2 M P a$ and $\sigma_{T C}=25 M P a$ with $\eta=4.91$, which result in $\alpha=0.121$ and $\gamma=2.370 . \beta$ is subjected to changes during the loading process with initial value of $\beta=7.667$. Parameters $m_{1}=1.0$ and $m_{2}=0.5$, so the asymptotes for the plastic potential are associated to the tensile and compression meridian, accordingly. $m_{3}=0.5$ and $m_{4}=1000$, which yields the initial values of $\alpha_{1}=1.614, \beta_{1}=3.791 \mathrm{MPa}$ and $\beta_{2}=11.139 \mathrm{MPa}$, as well as $\alpha_{2}=1.389$ and $A=8.349 \cdot 10^{-3} \mathrm{MPa}$. The modulus of the assumed linear hardening is $E_{P}=5 \mathrm{GPa}$ and the values of parameters describing damage are $H_{C}=120, H_{T}=120, \omega_{C}=0.1$ and $\omega_{T}=1$ [2]. For the original CDP model $\beta_{M}=0.1, \phi=30^{\circ}$ is assumed.

Obtained results of the numerical computations show consistency with the test results. For decreasing $\sigma_{X X}, \varepsilon_{X X}$ declines and the increase of $\varepsilon_{Y Y}=\varepsilon_{Z Z}$ is observed (Fig. 1(b),(c)) which is the outcome of the assumed constitutive law. The development of irreversible deformation begins in the first cycle of loading. Plastic components of the strain tensor become to dominate over elastic parts as the number of cycles grows, see Fig. 1(b). The relative volume (volumetric strain) grows for the initial stages of analysis but then it decreases (Fig. 1(d)).

The damage parameter $d$ grows during the plastic flow, see Fig. 1(e). Its development is responsible for the change of initial Young modulus, which is depicted on Fig. 1(c). $d$ reaches the value of 0.25 , which is usually near the point of complete destruction of material.

Plastic dissipation during the process is controlled to ensure thermodynamic consistency of the model. In the presented analysis dissipation is positive and increases as the yielding advances, see Fig. 1(f).

The difference between the original and the new potential and influence of parameters $m_{1}, m_{2}, m_{3}$ and $m_{4}$ of the proposed potential are analyzed for monotonic uniaxial compression and tension tests, where $\sigma_{X X}$ is imposed as shown on Fig. 2.

For both tests, regardless of $m_{i}$ selection, the $\varepsilon_{X X}$ component of strain tensor remains unchanged. It also matches the result for the CDP model. Furthermore, the modification of 
potential does not seem to influence damage parameter, which is only slightly different between models and does not vary for distinct values of $m_{i}$.

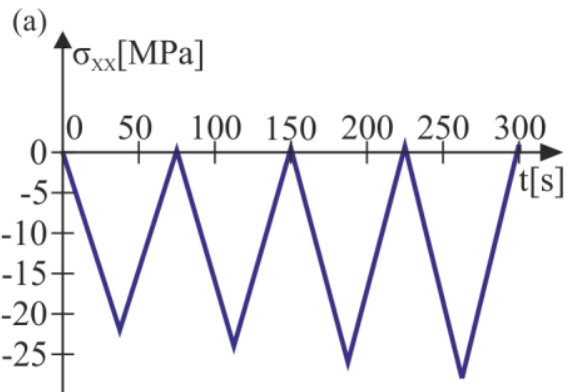

(c)
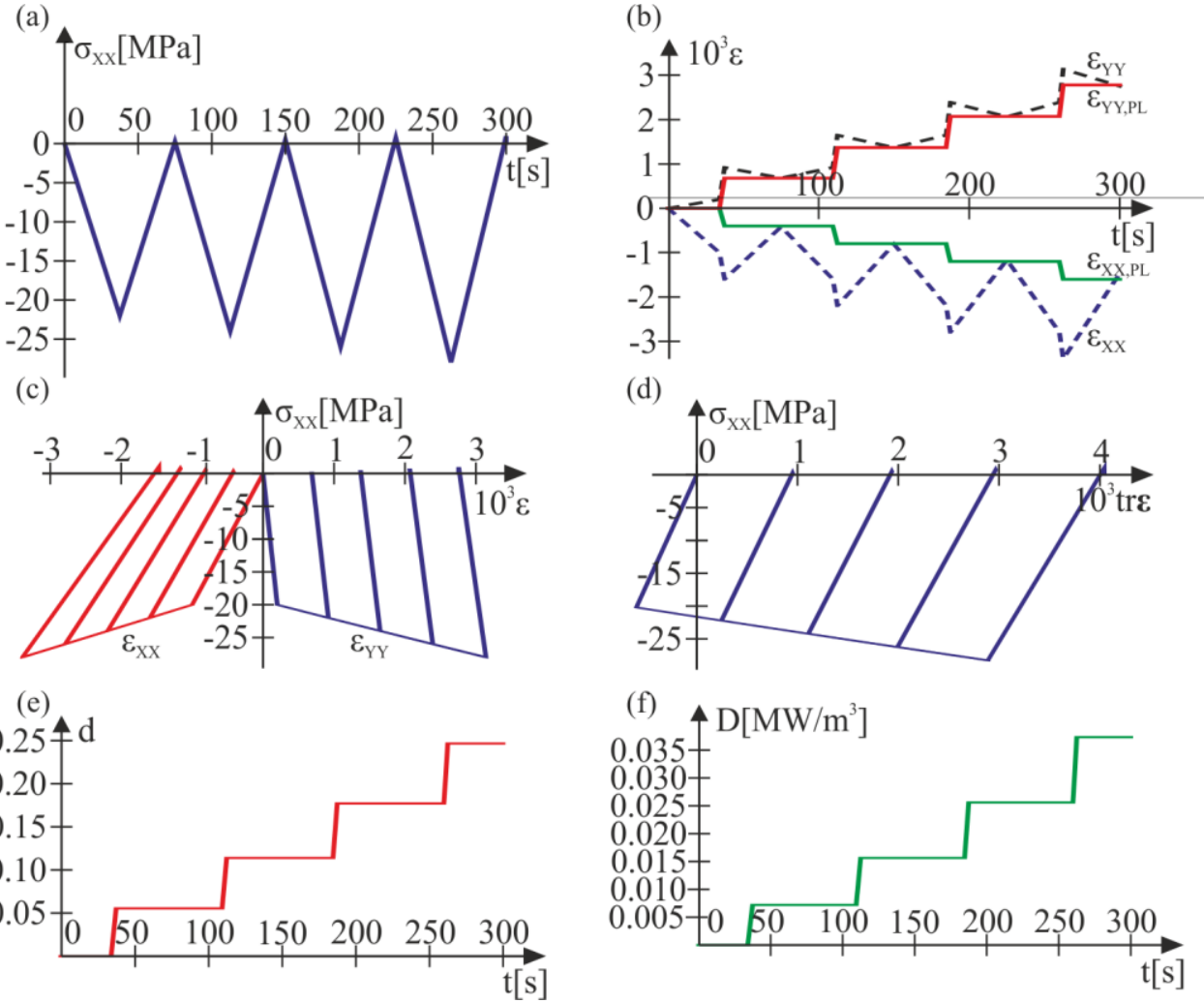

Fig. 1. Uniaxial cyclic test: (a) loading, (b) components of plastic and total strain tensor versus time, (c) components of strain tensor versus $\sigma_{X X}$, (d) volumetric strain versus $\sigma_{X X}$, (e) evolution of damage parameter, (f) specific dissipation.

(a)

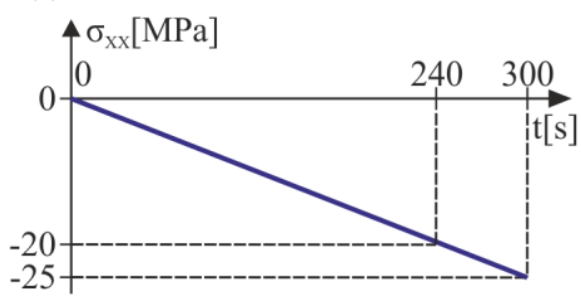

(b)

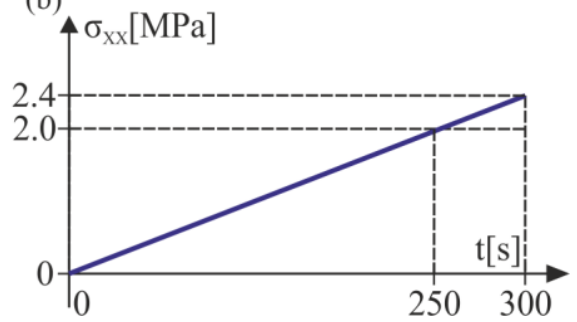

Fig. 2. Loading program in monotonic uniaxial tests: (a) compression, (b) tension.

For uniaxial compression test, the difference between original potential and new potential for variuos values of $m_{1}$ and $m_{2}$ is depicted on Fig. 3. An essential dissimilarity is observed in values of the lateral strain $\varepsilon_{Y Y}=\varepsilon_{Z Z}$, which implicates the difference of relative volume $\left(\varepsilon_{V}=\operatorname{tr} \varepsilon\right)$. As it is shown on Fig. 2(a), incerease of $m_{1}$ implicates growth of $\varepsilon_{V}$, while increase of $m_{2}$ causes $\varepsilon_{V}$ to decrease (Fig. 2(b)). Parameters $m_{1}$ and $m_{2}$ govern the slope of the plastic potential's asymptotes, so their great impact on computation results is apparent. However, $m_{2}$ affects plastic strain level stronger than $m_{1}$, because it controlls 
the compressive surface, where the considered test is located. Plastic lateral strain (or $\varepsilon_{V}$ ) for the CDP model is appreciably smaller than for the modified model.
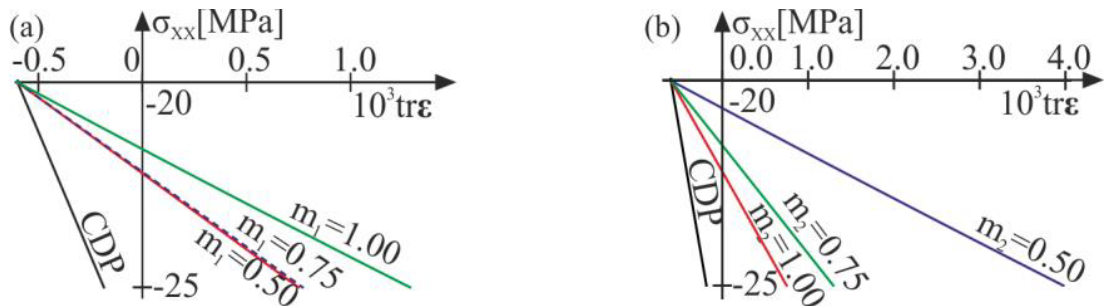

Fig. 3. Influence of values of parameters: (a) $m_{1}$ and (b) $m_{2}$ on relative volume ( $\varepsilon_{V}=\operatorname{tr} \varepsilon$ ) in the uniaxial compression test after crossing yield limit for: $\sigma_{C 0}=20 M P a, \sigma_{T 0}=2 M P a$, $\sigma_{B C}=23 M P a, \eta \sigma_{T C}=123 M P a, m_{1}=0.5, m_{2}=1.0, m_{3}=0.5, m_{4}=1$.

For the uniaxial compression test parameters $m_{3}$ and $m_{4}$ do not have any noticeable impact on the obtained results. The lack of influence is easily explicable, for the parameters control mainly the region near the potential's apex, whereas the test is located on compression cone.
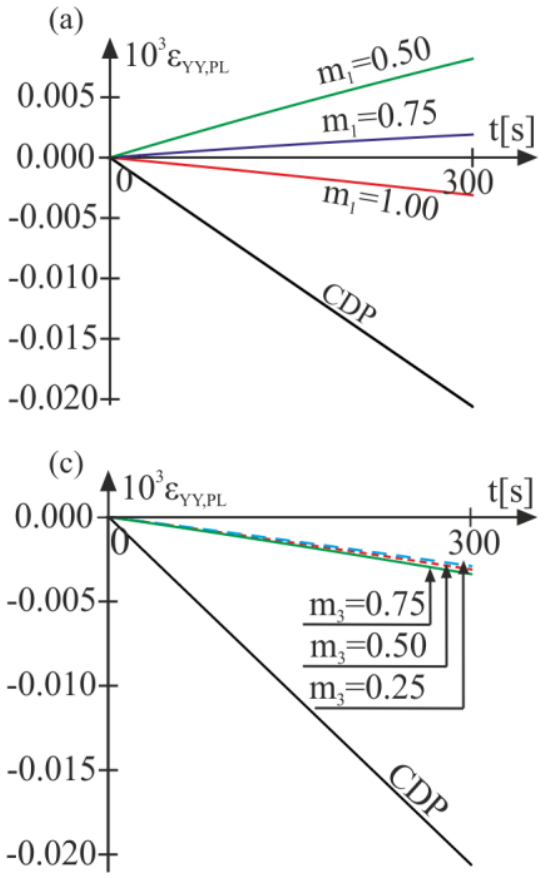
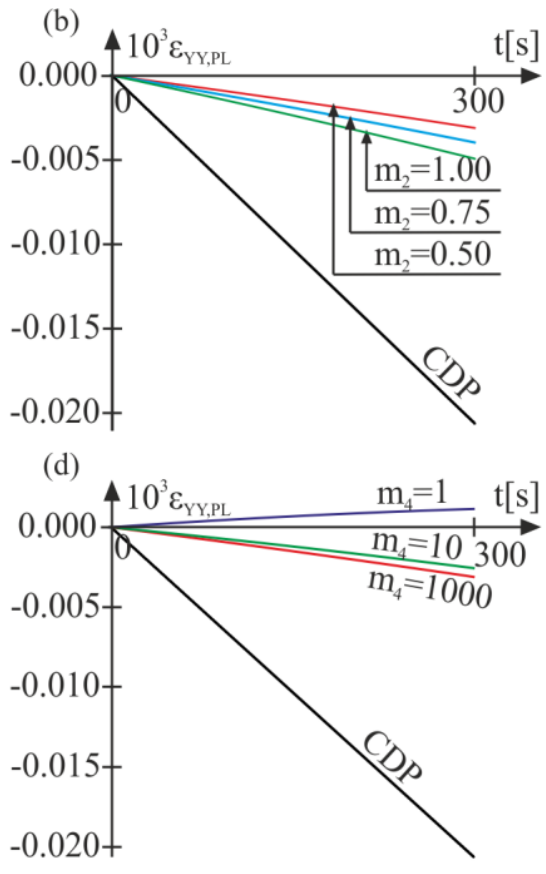

Fig. 4. Influence of selection of: (a) $m_{1}$, (b) $m_{2}$, (c) $m_{3}$ and (d) $m_{4}$ on the lateral components of plastic strain $\left(\varepsilon_{Y Y, P L}=\varepsilon_{Z Z, P L}\right)$ in the uniaxial tension test; $\sigma_{C 0}=20 M P a, \sigma_{T 0}=2 M P a$, $\sigma_{B C}=23 M P a, \eta \sigma_{T C}=123 M P a, m_{1}=1.0, m_{2}=0.5, m_{3}=0.5, m_{4}=1000$.

The results of uniaxial tension test are depicted on Fig. 4. It is shown that they depend on $m_{1}, m_{2}$ and $m_{4}$, while the influence of $m_{3}$ is negligible, compare Fig. 4(c). For lower values of $m_{1}$ (Fig. 4(a)) and low values of $m_{4}$ (Fig. 4(d)) negative lateral component of plastic stress is obtained which implicates that one should choose the tensile meridian to 
calibrate tension surface of potential $\left(m_{1}=1\right)$ and to select the potential meridians to be as close as possible to asymptotes (large values of $m_{4}$ ), compare Part I [1]. With the increase of $m_{2}$ lateral plastic strain becomes smaller, but the influence of $m_{2}$ on the result is appreaciably smaller than for $m_{1}$ and $m_{4}, \varepsilon_{V}$ grows during the loading process. The difference between the new and original potential is noticeable, regardless of allowable change of $m_{i}$ parameters.

The considerable differences between curves for variuos values of parameters enable to calibrate $m_{1}$ and $m_{4}$ on basis of the uniaxial tension test and $m_{2}$ on grounds of the uniaxial compression experiment.

Assumed values of the material parameters are chosen arbitrarily to depict typical properties of concrete. To reflect properties of particular type of concrete and prove quantitative compatibility of the model it is necessary to propose a proper calibration procedure.

Strain-stress curves for concrete subjected to uniaxial load include decreasing branch not shown in the simulation. It is possible to model material behavior in this area assuming an appropriate law of hardening (softening) instead of (7).

\section{Conclusions}

The results of a simple simulation of the uniaxial cyclic test prove that the proposed model properly reflects basic features of material behavior, although it is necessary to perform more complex numerical computations to ensure its qualitative and quantitative compatibility with the observed results of experiments.

Another important issue to be addressed is the calibration of the free parameters included in the definition of potential. A preliminary analysis shows that the uniaxial tests results are affected mainly by $m_{1}, m_{2}$ and $m_{4}$. As for $m_{3}$, another test should be performed to determine its advisable value, preferebly located in the triaxial tension zone.

Extended flexibility of the presented potential allows for more accurate calibration of the material response. This feature is desirable when considering structures where material failure can occur both by tension and compression in some regions. Original Abaqus CDP model cannot cover such cases correctly.

\section{References}

1. A. Szwed, I. Kamińska, Part I - accompanying paper

2. Abaqus Theory Manual

3. www.wolfram.com

4. S. Murakami, Continuum Damage Mechanics (Springer, 2012)

5. J. Lubliner, J. Oliver, S. Oller, E. Onate, Int. J. Solids Struct. 25(3), 299 (1989)

6. J. Lee, G. Fenves, Earthquake Eng. Struct. Dyn. 27, 937 (1998)

7. I. Kamińska, A. Szwed, Concrete and concrete structure - experiments, E. Szmigiera, P. Łukowski, S. Jemioło (eds.), 93 (OWPW, Warszawa, 2015) (in Polish)

8. P. Kmiecik, M. Kamiński, Archives of Civil and Mechanical Engineering XI(3), 623 (2011)

9. M. Szczecina, A. Winnicki, ASEM15 (Korea, 2015)

10. A. Truty, T. Zimmermann, Numerics in Geotechnics and Structures 1 (2015) 